Numerical Calculation of the Spectrum of the Severe (1\%) Lighting Current and Its First Derivative

C. G. Brown, M. M. Ong, M. P. Perkins, R. D. Speer

February 16, 2010 
This document was prepared as an account of work sponsored by an agency of the United States government. Neither the United States government nor Lawrence Livermore National Security, LLC, nor any of their employees makes any warranty, expressed or implied, or assumes any legal liability or responsibility for the accuracy, completeness, or usefulness of any information, apparatus, product, or process disclosed, or represents that its use would not infringe privately owned rights. Reference herein to any specific commercial product, process, or service by trade name, trademark, manufacturer, or otherwise does not necessarily constitute or imply its endorsement, recommendation, or favoring by the United States government or Lawrence Livermore National Security, LLC. The views and opinions of authors expressed herein do not necessarily state or reflect those of the United States government or Lawrence Livermore National Security, LLC, and shall not be used for advertising or product endorsement purposes.

This work performed under the auspices of the U.S. Department of Energy by Lawrence Livermore National Laboratory under Contract DE-AC52-07NA27344. 


\section{Numerical Calculation of the Spectrum of the Severe (1\%) Lighting Current and Its First Derivative}

Charles G. Brown Jr., Mike M. Ong, Michael P. Perkins, and Ron D. Speer

August 17, 2010

\section{Contents}

1 Introduction 1

$2 \quad 1 \%$ Lightning Current and Its First Derivative 2

3 Numerical Method for Calculating the Spectra 2

4 Test Using 1\% Double-Exponential Waveform 6

5 Spectra of the 1\% Lightning Current Waveforms and First Derivatives

\section{Introduction}

Recently, the direct-strike lighting environment for the stockpile-to-target sequence was updated [1]. In [1], the severe (1\%) lightning current waveforms for first and subsequent return strokes are defined based on Heidler's waveform. This report presents numerical calculations of the spectra of those $1 \%$ lightning current waveforms and their first derivatives. First, the $1 \%$ lightning current models are repeated here for convenience. Then, the numerical method for calculating the spectra is presented and tested. The test uses a double-exponential waveform and its first derivative, which we fit to the previous $1 \%$ direct-strike lighting environment from [2]. Finally, the resulting spectra are given and are compared with those of the double-exponential waveform and its first derivative. 


\section{$2 \quad 1 \%$ Lightning Current and Its First Derivative}

The updated waveform ${ }^{1}$ of the lighting current is [1]

$$
I(t)=\sum_{k=1}^{m} \frac{I_{0_{k}}}{\eta_{k}} e^{-t / \tau_{2 k}} \frac{\left(\frac{t}{\tau_{1_{k}}}\right)^{n_{k}}}{1+\left(\frac{t}{\tau_{1_{k}}}\right)^{n_{k}}}
$$

with

$$
\eta_{k}=e^{-\frac{\tau_{1_{k}}}{\tau_{2_{k}}}\left(n_{k} \frac{\tau_{2_{k}}}{\tau_{1_{k}}}\right)^{1 / n_{k}}}
$$

The updated waveform of the first derivative of the lightning current is [1]

$$
\frac{d I(t)}{d t}=\sum_{k=1}^{m} \frac{I_{0_{k}}}{\eta_{k} \tau_{2_{k}}} \frac{e^{-t / \tau_{2_{k}}\left(\frac{t}{\tau_{1_{k}}}\right)^{n_{k}}\left[t-n_{k} \tau_{2_{k}}+t\left(\frac{t}{\tau_{1_{k}}}\right)^{n_{k}}\right]}}{t\left[1+\left(\frac{t}{\tau_{1_{k}}}\right)^{n_{k}}\right]^{2}}
$$

The parameter values used in Eqns. 1 and 3 for median (50\%) negative first and subsequent strokes are given in Tables 1 and 2, respectively. In order to obtain 1\% lighting currents and first derivatives, the resulting $50 \%$ waveforms are multiplied by the values in Table 3 . We use the scaling factors in Table 3 instead of multiplying by five as suggested in [1] in order to better match the $1 \%$ peak current and peak rise rate from [1]. Note that for the $1 \%$ first return stroke peak current we use $200 \mathrm{kA}$ instead of $150 \mathrm{kA}$ to roughly approximate a frequency-weighted average of the negative and positive peak currents. Figures 1 and 2 show overview and expanded plots of the $1 \%$ first return stroke $I(t)$ and $d I(t) / d t$, respectively. Figures 3 and 4 display $I(t)$ and $d I(t) / d t$, respectively, for the $1 \%$ subsequent return stroke. We wish to compute the spectra of these functions. (Figures 1, 2, 3, and 4 also include the 1\% double-exponential waveforms for comparison. See Section 4 for descriptions of the waveforms.)

\section{Numerical Method for Calculating the Spec- tra}

Calculating the spectra of $I(t)$ and $d I(t) / d t$ is performed numerically using the Fast Fourier Transform (FFT), rather than analytically. Let the FFT of the length $N$ discrete-time signal $x[n]$ be defined ${ }^{2}$ as $[4,5]$

\footnotetext{
${ }^{1}$ For numerical reasons we use an alternate but equivalent expression for the fraction in Eqn. 1: $\left.\left({ }^{t} / \tau_{1_{k}}\right)^{n_{k}} /{ }_{1+\left({ }^{t} / \tau_{1_{k}}\right.}\right)^{n_{k}}=1 / 1+\left(t / \tau_{1_{k}}\right)^{-n_{k}}$

${ }^{2}$ Actually, Eqn. 4 is the definition of the Discrete Fourier Transform (DFT), and the FFT is a fast algorithm for computing the DFT [3, 4, 5]. We call Eqn. 4 the FFT for convenience.
} 
Table 1: Parameter values used in Eqn. 1 for median (50\%) negative first return strokes [1].

\begin{tabular}{lllll}
\hline$k$ & $I_{0 k}(\mathrm{kA})$ & $n_{k}$ & $\tau_{1 k}(\mu \mathrm{s})$ & $\tau_{2 k}(\mu \mathrm{s})$ \\
\hline 1 & 3 & 2 & 15 & 30 \\
2 & 3 & 3 & 15 & 30 \\
3 & 3 & 9 & 20 & 30 \\
4 & 3 & 11 & 20 & 30 \\
5 & 25 & 150 & 10 & 23 \\
6 & 15 & 2 & 30 & 250 \\
\hline
\end{tabular}

Table 2: Parameter values used in Eqn. 1 for median (50\%) negative subsequent return strokes [1].

$$
\begin{gathered}
\begin{array}{lllll}
\hline k & I_{0 k}(\mathrm{kA}) & n_{k} & \tau_{1 k}(\mu \mathrm{s}) & \tau_{2 k}(\mu \mathrm{s}) \\
\hline 1 & 15 & 5 & 0.2 & 50 \\
\hline
\end{array} \\
F F T\{x[n]\}=X[k]=\sum_{n=0}^{N-1} x[n] e^{-j 2 \pi k \frac{n}{N}} ; k=0, \ldots, N-1
\end{gathered}
$$

where $j$ denotes the square root of negative one. Suppose we wish to compute the spectrum of a continuous-time signal $x(t)$. One method for approximating the spectrum is as follows $[4,5,6] .{ }^{3}$ First, we sample $x(t)$ at $f_{s}$ samples per second to produce the length $N$ array $x[n]$ :

$$
x[n]=x\left(n / f_{s}\right) ; n=0, \ldots, N-1
$$

Then, the continuous-time Fourier transform of $x(t)$

$$
\mathcal{F}\{x(t)\}=\int_{-\infty}^{+\infty} x(t) e^{-j 2 \pi f t} d t
$$

can be approximated as

\footnotetext{
${ }^{3}$ Another more accurate method is in [7].
}

Table 3: Scaling values for severe (1\%) return strokes. Multiply Eqns. 1 and 3 , with parameters from Tables 1 and 2 , by the scaling values to yield the $1 \%$ waveforms.

\begin{tabular}{lll}
\hline $1 \%$ lightning cases covered by scaling values & $I(t)$ & $d I(t) / d t$ \\
\hline Negative and positive first return stroke & 5.98 & 4.18 \\
Negative subsequent return stroke & 3.33 & 4.0505 \\
\hline
\end{tabular}



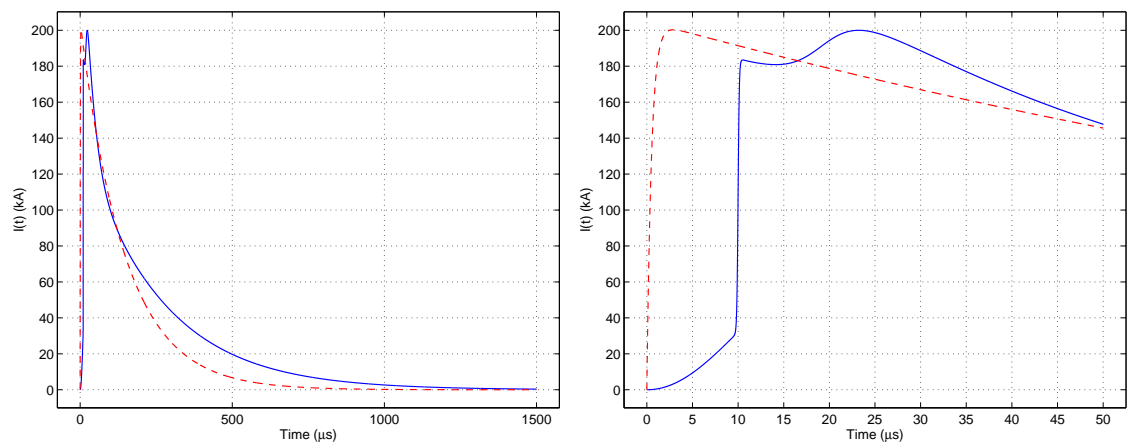

Figure 1: $1 \%$ first return stroke current (blue) and 1\% double-exponential current (red dashed). The $1 \%$ first return stroke current is computed by multiplying Eqn. 1, with parameters from Table 1, by the scaling factor in Table 3. The plot on the right is an expanded view of the one on the left.

$$
\mathcal{F}\{x(t)\} \approx \Delta t F F T\{x[n]\}=F F T\{x[n]\} / f_{s}
$$

where $\mathcal{F}\{x(t)\}$ denotes the Fourier transform of $x(t)$. FFT $\{x[n]\}$ represents the FFT of $x[n]$ as defined in Eqn. 4, and

$$
\Delta t=1 / f_{s}
$$

is the sampling interval.

Since $x[n]$ is real, $F F T\{x[n]\}$ exhibits complex conjugate symmetry $[3,6,8$, 9]. Thus, we take the subset $k=0, \ldots, N^{\prime}-1$ of $F F T\{x[n]\}$, with $N^{\prime}$ given as

$$
N^{\prime}=\left\{\begin{array}{cl}
N / 2 & \text { if } \mathrm{N} \text { is even } \\
N / 2-1 / 2 & \text { if } \mathrm{N} \text { is odd }
\end{array}\right.
$$

which corresponds to the positive frequencies $k \Delta f$ for $k=0, \ldots, N^{\prime}-1$, with

$$
\Delta f=\frac{1}{N \Delta t}=\frac{f_{s}}{N}
$$

Interpolation to a finer frequency sampling can be achieved by appending zeros to $x[n]$ to a length $N_{z p}[4,5]$. The new frequency spacing is then $\Delta f_{z p}=$ $f_{s} / N_{z p}$, and Eqns. 7 and 9 are adjusted as follows:

$$
\begin{gathered}
\mathcal{F}\{x(t)\} \approx \Delta t F F T\left\{x[n]_{z p}\right\}=F F T\left\{x[n]_{z p}\right\} / f_{s} \\
N_{z p}^{\prime}=\left\{\begin{array}{cl}
N_{z p} / 2 & \text { if } \mathrm{N}_{z p} \text { is even } \\
N_{z p} / 2-1 / 2 & \text { if } \mathrm{N}_{z p} \text { is odd }
\end{array}\right.
\end{gathered}
$$

The frequencies corresponding to the samples in Eqn. 12 are $k \Delta f_{z p}$ for $k=$ $0, \ldots, N_{z p}^{\prime}-1$. Note that zero padding results in interpolation to a finer frequency 

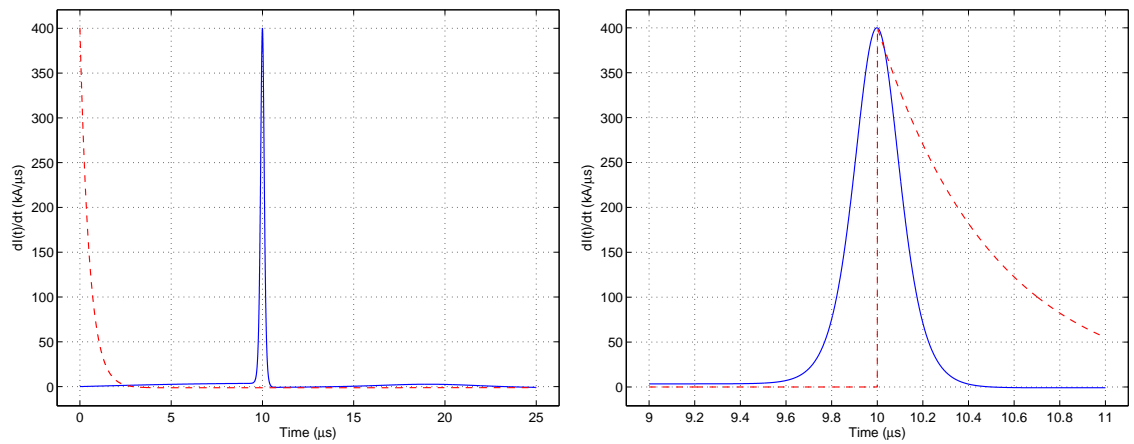

Figure 2: $1 \%$ first return stroke current first derivative (blue) and $1 \%$ doubleexponential current first derivative (red dashed). The $1 \%$ first return stroke current first derivative is computed by multiplying Eqn. 3, with parameters from Table 1, by the scaling factor in Table 3. The plot on the right is an expanded view of the one on the left, with the $1 \%$ double-exponential current first derivative shifted to the right by $10 \mu \mathrm{s}$.

sampling without an actual increase in frequency resolution; a longer record of non-zero samples of $x[n]$ is required to truly increase the frequency resolution [5].

For a well-behaved $x(t)$, if $x(t)$ is also approximately time- and band-limited for the sampling defined in Eqn. 5, then Eqn. 7 is a reasonable approximation of the Fourier transform of $x(t)[4,5,6]{ }^{4}$ In order to satisfy the time-limited criterion we must sample $I(t)$ and $d I(t) / d t$ long enough so that they are approximately zero, thus minimizing ripple on the spectrum $[5,6] .^{5}$ The ripple is a result of truncation, and if $x(t)$ were truly time-limited, the only errors would be due to aliasing [6]. The band-limited criterion requires us to sample at a large enough $f_{s}$ so that aliasing is below our desired level of accuracy [6] for the frequency range in which we are interested. According to [6] the "computation of the Fourier transform only requires that we exercise care in the choice of $\left[1 / f_{s}\right]$ and $N$ and interpret the results correctly."

We can approach satisfying the criteria of being approximately time- and band-limited by performing a simple convergence study for different values of $f_{s}$ and $T$, where $T=N / f_{s}$. We use this approach in Sections 4 and 5 to obtain converged spectra.

\footnotetext{
${ }^{4}$ For more detailed discussions on the pitfalls of using the FFT to approximate the Fourier transform, see Refs. $[4,5,6]$.

${ }^{5}$ We could also use a one-sided tapered window, with a step function at the beginning and the taper at the end, but that would bias the spectrum.
} 

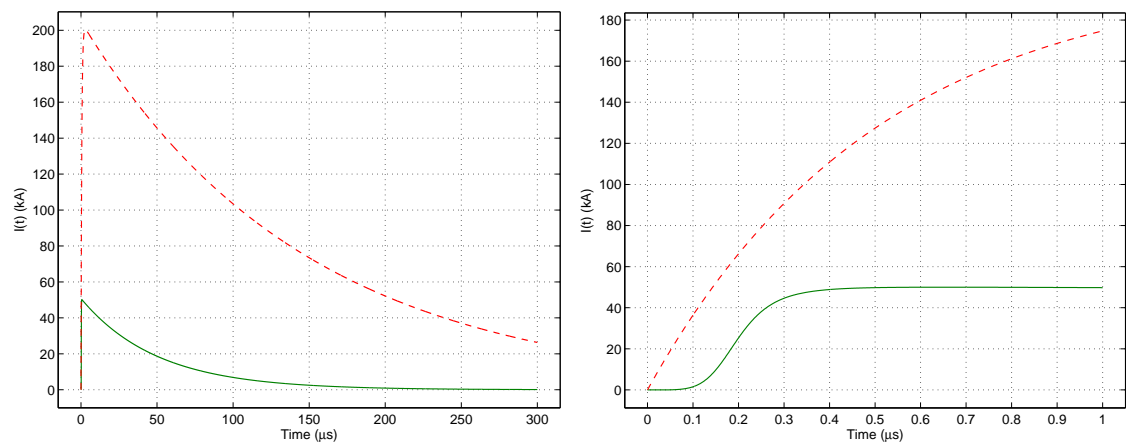

Figure 3: $1 \%$ subsequent return stroke current (green) and 1\% doubleexponential current (red dashed). The $1 \%$ subsequent return stroke current is computed by multiplying Eqn. 1, with parameters from Table 1, by the scaling factor in Table 3 . The plot on the right is an expanded view of the one on the left.

\section{Test Using 1\% Double-Exponential Waveform}

Before we apply the above method to approximate the spectra of the $1 \%$ lightning current models and their derivatives from [1], we first test the method using the previous $1 \%$ lightning current environment parameters [2]. The following double-exponential return stroke current model approximates the peak current $(200 \mathrm{kA})$, peak rise rate $(400 \mathrm{kA} / \mu \mathrm{s})$, total charge transferred $(29.8 \mathrm{C})$, and action integral $\left(3 \times 10^{6} \mathrm{~A}^{2} \mathrm{~s}\right)$ for the previous $1 \%$ lightning current in [2]:

$$
I(t)=I_{0}\left[e^{-t / \tau_{f w h m}}-e^{-t / \tau_{\text {rise }}}\right]
$$

where $\tau_{f w h m} \approx 146.2 \mu \mathrm{s}$ is the full-width half-maximum time, $\tau_{\text {rise }} \approx 0.51 \mu \mathrm{s}$ is the rise time, and $I_{0} \approx 205 \mathrm{kA}$. The first derivative is

$$
\frac{d I(t)}{d t}=\frac{I_{0}}{\tau_{\text {fwhm }} \tau_{\text {rise }}}\left[\tau_{\text {fwhm }} e^{-t / \tau_{\text {rise }}}-\tau_{\text {rise }} e^{-t / \tau_{f w h m}}\right]
$$

The double-exponential current model is especially useful for testing since its Fourier transform can be determined analytically [10]:

$$
\mathcal{F}\{I(t)\}=I(f)=\frac{I_{0}\left(\tau_{f w h m}-\tau_{\text {rise }}\right)}{\tau_{\text {fwhm }} \tau_{\text {rise }}\left(j 2 \pi f+\frac{1}{\tau_{f w h m}}\right)\left(j 2 \pi f+\frac{1}{\tau_{\text {rise }}}\right)}
$$

The Fourier transform of the first derivative of the double-exponential lightning current can also be written in closed form:

$$
\mathcal{F}\{d I(t) / d t\}=I^{\prime}(f)=j 2 \pi f I(f)=\frac{j 2 \pi f I_{0}\left(\tau_{\text {fwhm }}-\tau_{\text {rise }}\right)}{\tau_{f w h m} \tau_{\text {rise }}\left(j 2 \pi f+\frac{1}{\tau_{\text {fwhm }}}\right)\left(j 2 \pi f+\frac{1}{\tau_{\text {rise }}}\right)}
$$



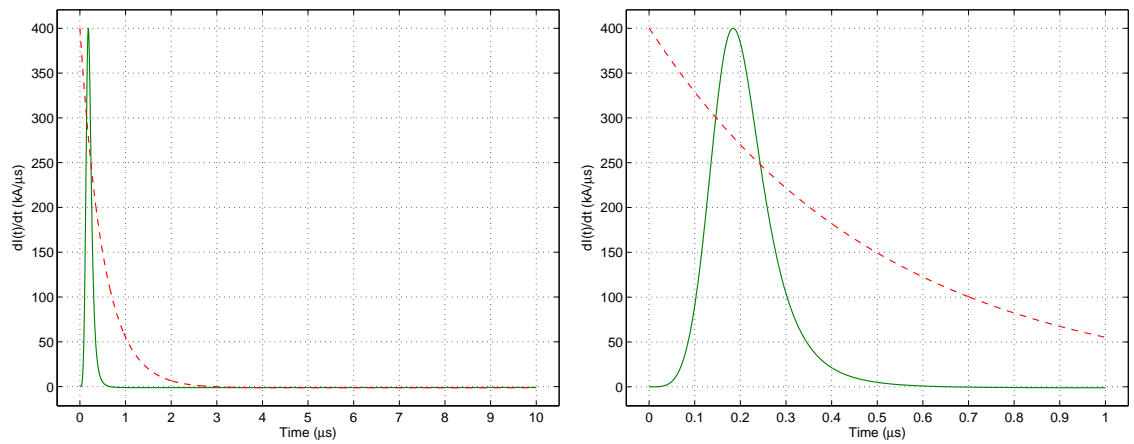

Figure 4: $1 \%$ subsequent return stroke current first derivative (blue) and $1 \%$ double-exponential current first derivative (red dashed). The $1 \%$ subsequent return stroke current first derivative is computed by multiplying Eqn. 3, with parameters from Table 1, by the scaling factor in Table 3 . The plot on the right is an expanded view of the one on the left.

See Figures 1, 2, 3, and 4 in Section 2 for plots of the double-exponential functions.

Our goal is to achieve an approximation within $\pm 0.5 \mathrm{~dB}$ in magnitude and $\pm 0.5^{\circ}$ in phase over the frequency range from 0 to $10 \mathrm{MHz} .{ }^{6}$ First, we plot the analytical transform (Eqn. 15) on the same plot with the approximation (Eqn. 7) with a reasonable $T$ and four $f_{s}$ values. In this case we start with $T=1.25 \mathrm{~ms}$ and use $f_{s}=20,40,80,160 \mathrm{MHz}$. Figure 5 shows the convergence of the magnitude and the phase separately. Figure 6 is an expanded view of Fig. 5 showing that the magnitude converges to the analytical value from above. The phase converges to the analytical value from below. Note the ripples, due to truncation, in both magnitude and phase. The zero-frequency (DC) magnitude values are only $0.0018 \mathrm{~dB}$ below the analytical value. The phase values are consistent with the fact that the DC value is always real. Increasing $T$ to $T=2 \mathrm{~ms}$ reduces the ripples further (Figs. 7 and 8 ). The error plot in Fig. 9 provides a better depiction of the accuracy of the approximate transform for $T=2 \mathrm{~ms}$. The approximate transform is now clearly within $\pm 0.5 \mathrm{~dB}$ in magnitude and $\pm 0.5^{\circ}$ in phase, even for $f_{s}=80 \mathrm{MHz}$.

\footnotetext{
${ }^{6}$ The magnitude and phase goals that we use here and in the rest of this report are arbitrary and are selected to be reasonably small and achievable.
} 

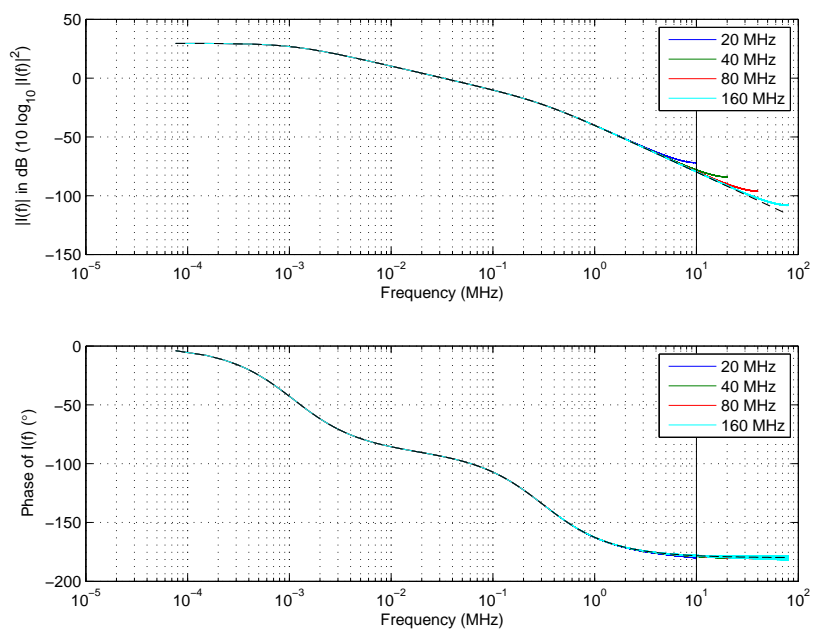

Figure 5: Convergence of the magnitude and phase of the approximate transform of the $1 \%$ double-exponential lightning current for $T=1.25 \mathrm{~ms}$. The black dashed line is the analytical transform. The vertical solid black line marks $10 \mathrm{MHz}$, which is the highest frequency of interest in this test case.
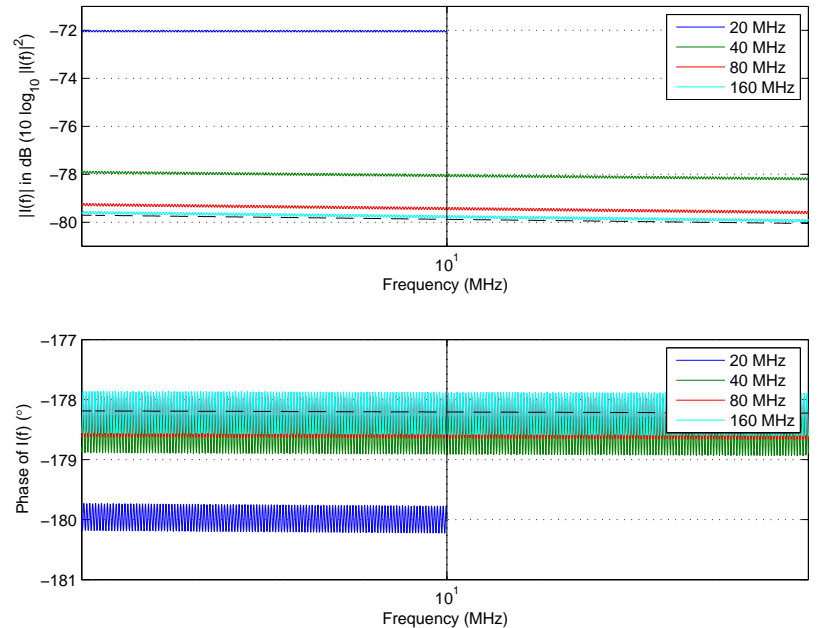

Figure 6: Expanded view of Fig. 5 around $10 \mathrm{MHz}$. 

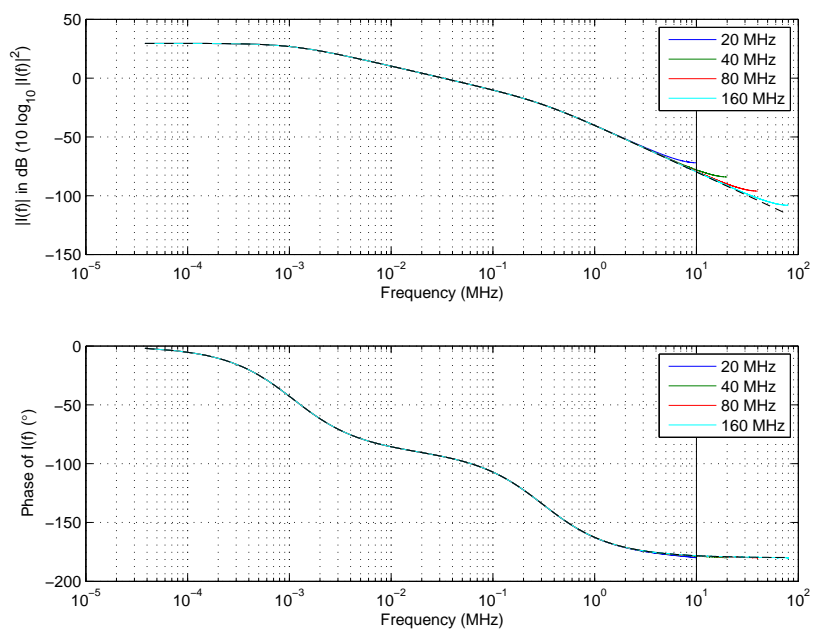

Figure 7: Convergence of the magnitude and phase of the approximate transform of the $1 \%$ double-exponential lightning current for $T=2 \mathrm{~ms}$. The black dashed line is the analytical transform. The vertical solid black line marks $10 \mathrm{MHz}$, which is the highest frequency of interest in this test case.
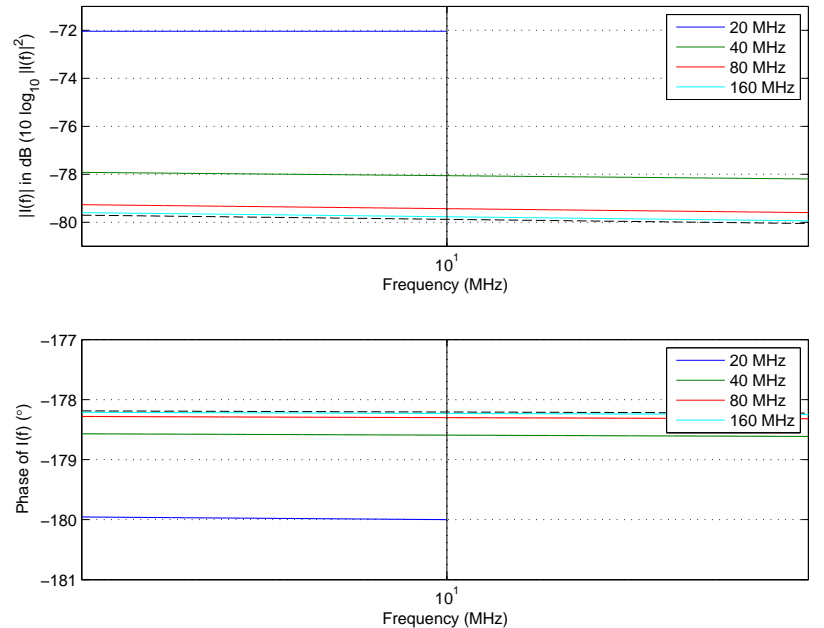

Figure 8: Expanded view of Fig. 7 around $10 \mathrm{MHz}$. 

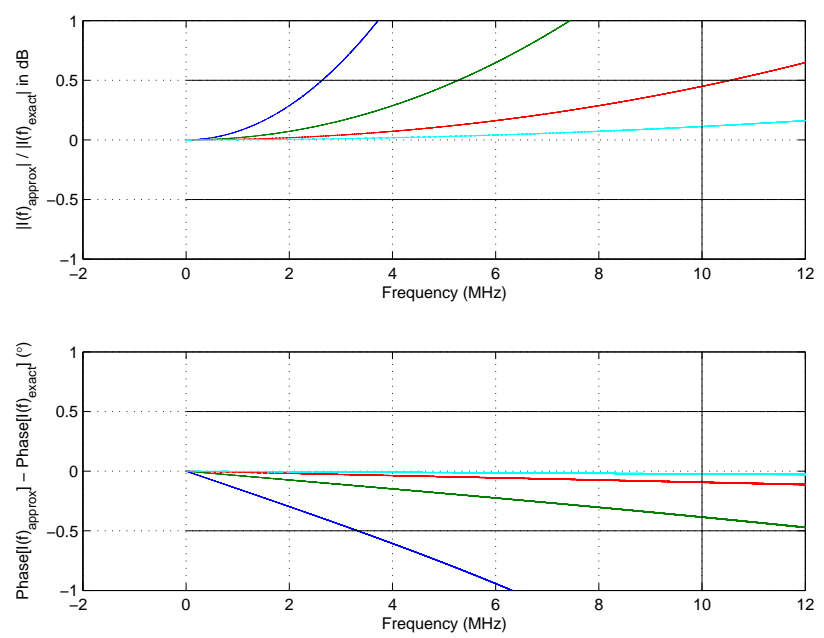

Figure 9: Errors in the magnitude and phase of the approximate (approx) transform of the $1 \%$ double-exponential lightning current for $T=2 \mathrm{~ms}$ relative to the analytical (exact) transform. The vertical solid black line marks $10 \mathrm{MHz}$, which is the highest frequency of interest in this test case. The horizontal black lines mark the $\pm 0.5 \mathrm{~dB}$ and $\pm 0.5^{\circ}$ limits. The color scheme for the sampling frequencies is the same as in Figs. 5, 6, 7, and 8.

Next, for the first derivative we plot the analytical transform (Eqn. 16) on the same plot as the approximation (Eqn. 7) with a reasonable $T$ and six $f_{s}$ values: $T=0.5 \mathrm{~ms}$ and $f_{s}=40,80,160,320,640 \mathrm{MHz}$. Figure 10 shows the convergence of the magnitude and the phase separately. Figure 11 is an expanded view of Fig. 10. Note that the magnitude at $10 \mathrm{MHz}$ is within the range of our goal; however, the phase is about $3^{\circ}$ off the analytical value at $10 \mathrm{MHz}$ even for the $640 \mathrm{MHz}$ case. Better values can be obtained for the first derivative by approximating the spectrum of the current and then multiplying by $j 2 \pi f$. Using this approach with $T=2 \mathrm{~ms}$ and $f_{s}=20,40,80,160 \mathrm{MHz}$ we see clear convergence (Figs. 12 and 13) with the same convergence properties as with the current. The error plot in Fig. 14 displays the accuracy of the approximate transform. As with the current, the approximate transform of the first derivative is now clearly within $\pm 0.5 \mathrm{~dB}$ in magnitude and $\pm 0.5^{\circ}$ in phase, even for $f_{s}=80 \mathrm{MHz}$. 

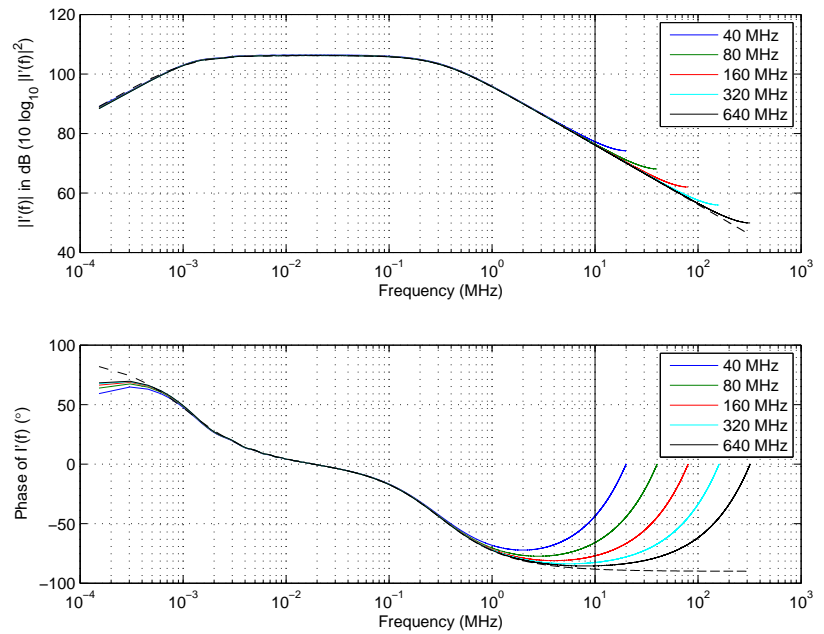

Figure 10: Convergence of the magnitude and phase of the approximate transform of the first derivative of the $1 \%$ double-exponential lightning current for $T=0.5 \mathrm{~ms}$. The black dashed line is the analytical transform. The vertical solid black line marks $10 \mathrm{MHz}$, which is the highest frequency of interest in this test case.
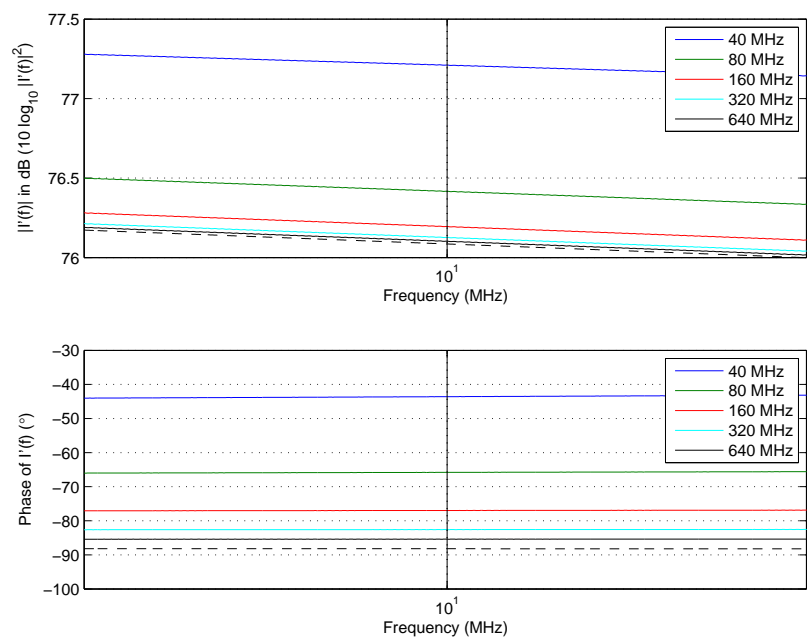

Figure 11: Expanded view of Fig. 10 around $10 \mathrm{MHz}$. 

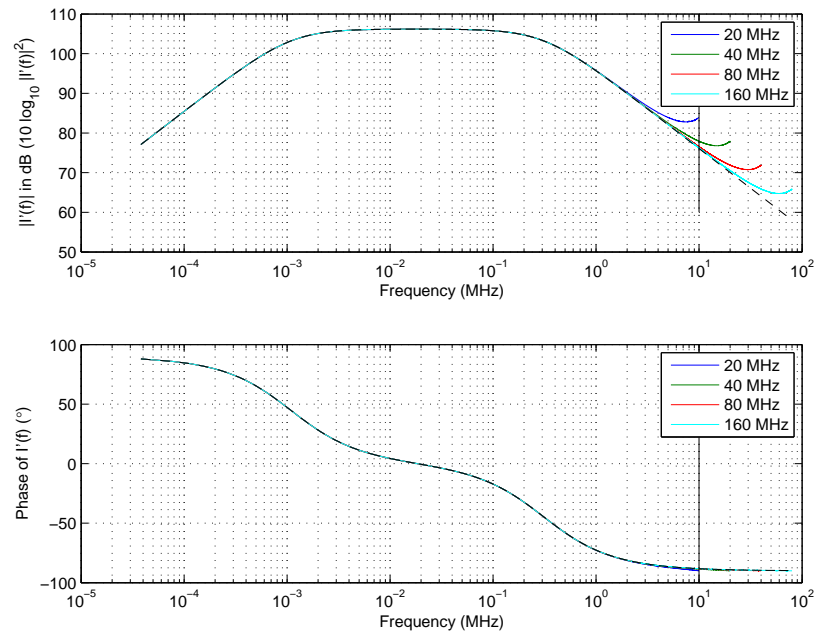

Figure 12: Convergence of the magnitude and phase of the approximate transform of the first derivative of the $1 \%$ double-exponential lightning current for $T=2 \mathrm{~ms}$. The black dashed line is the analytical transform. The vertical solid black line marks $10 \mathrm{MHz}$, which is the highest frequency of interest in this test case.
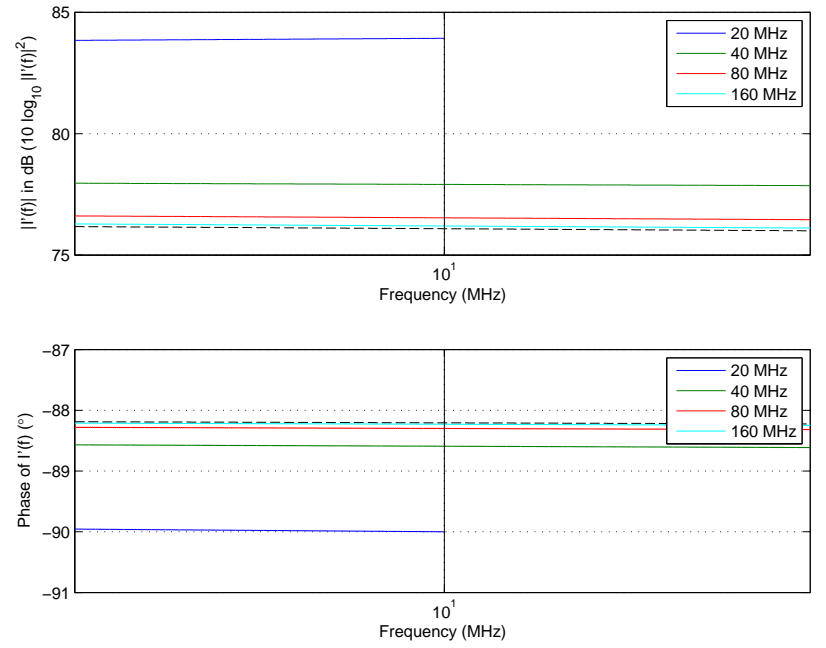

Figure 13: Expanded view of Fig. 12 around $10 \mathrm{MHz}$. 

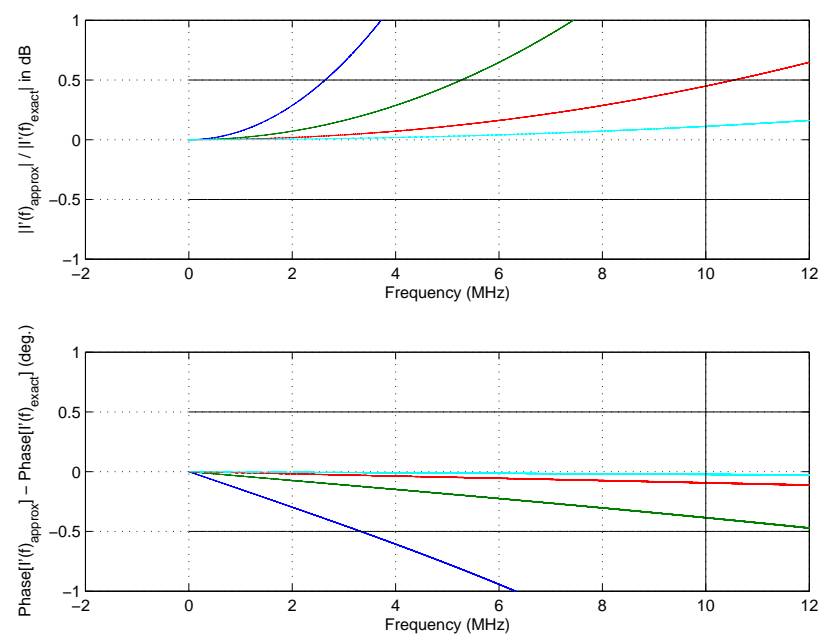

Figure 14: Errors in the magnitude and phase of the approximate (approx) transform of the first derivative of the $1 \%$ double-exponential lightning current for $T=2 \mathrm{~ms}$ relative to the analytical (exact) transform. The vertical solid black line marks $10 \mathrm{MHz}$, which is the highest frequency of interest in this test case. The horizontal black lines mark the $\pm 0.5 \mathrm{~dB}$ and $\pm 0.5^{\circ}$ limits. The color scheme for the sampling frequencies is the same as in Figs. 12 and 13.

\section{Spectra of the 1\% Lightning Current Wave- forms and First Derivatives}

We now apply the methods listed in Sections 3 and 4 to numerically approximate the Fourier transforms of $I(t)$ and $d I(t) / d t$ for the Heidler $1 \%$ first and subsequent return stroke cases. First, we approximate the Fourier transform of $I(t)$ for the Heidler $1 \%$ first return stroke current. Our initial sampling duration is $T=2 \mathrm{~ms}$, with $f_{s}=20,40,80,160 \mathrm{MHz}$. The results are shown in Fig. 15. The wrapped phase is taken after multiplying by $\exp (j 2 \pi f \cdot 10 \mu s)$, which corresponds to a time advance of $10 \mu \mathrm{s}$. Note the strange ripples just below $10 \mathrm{MHz}$. Increasing $T$ pushes this anomaly past the $10 \mathrm{MHz}$ mark. Figures 16 and 17 are plots of approximations over a range of $T(2,4,6,8 \mathrm{~ms})$ with a fixed $f_{s}=160 \mathrm{MHz}$. As in Fig. 15, the wrapped phase is taken after multiplying by $\exp (j 2 \pi f \cdot 10 \mu s)$. After $T=6 \mathrm{~ms}$ the anomaly decreases to a position just past $10 \mathrm{MHz}$. Up to $10 \mathrm{MHz}$ the magnitude is within $\pm 0.5 \mathrm{~dB}$. However, the phase is within $\pm 0.5^{\circ}$ up to only about $9 \mathrm{MHz}$. At $10 \mathrm{MHz}$ the phase is within about $\pm 1.5^{\circ}$. The observed behavior could indicate numerical issues, so we do not increase $T$ further. Perhaps higher-order approximations of the Fourier transform, such as in [7], and/or more accurate numerical evaluation of the Heidler 
lightning current expression could remove or validate the anomaly. Figures 18 and 19 display an additional convergence study using $f_{s}=20,40,80,160 \mathrm{MHz}$ with $T$ fixed at $6 \mathrm{~ms}$. The results indicate that parameter values $f_{s}=40 \mathrm{MHz}$ and $T=6 \mathrm{~ms}$ are sufficient for the Heidler first return stroke with a revised phase goal of about $\pm 1.5^{\circ}$.

The Heidler $1 \%$ subsequent return stroke is much more tractable than the first return stroke. First, we perform a convergence study for $T=6 \mathrm{~ms}$ and $f_{s}=20,40,80,160 \mathrm{MHz}$. See Figs. 20 and 21 . The wrapped phase is taken after multiplying by $\exp (j 2 \pi f \cdot 0.1991 \mu s)$ (a $0.1991 \mu$ s time advance). The parameter value $f_{s}=40 \mathrm{MHz}$ yields a spectrum within $\pm 0.5 \mathrm{~dB}$ in magnitude and $\pm 0.5^{\circ}$ in phase. A further convergence study with $f_{s}=40 \mathrm{MHz}$ and $T=0.2,0.4,0.8,1 \mathrm{~ms}$ indicates that the same level of convergence can be obtained with $f_{s}=40 \mathrm{MHz}$ and $T=0.8 \mathrm{~ms}$. Refer to Figs. 22 and 23 .

We obtain the first derivatives for the first and subsequent Heidler 1\% return strokes by multiplying the return stroke currents by $j 2 \pi f$. We use $T=6 \mathrm{~ms}$ for the first, $T=0.8 \mathrm{~ms}$ for the subsequent, and $f_{s}=40 \mathrm{MHz}$ for both. Figures 24 and 25 display the results. The wrapped phase is taken after multiplying by $\exp (j 2 \pi f \cdot 10 \mu s)$ or $\exp (j 2 \pi f \cdot 0.1991 \mu s)$, respectively.
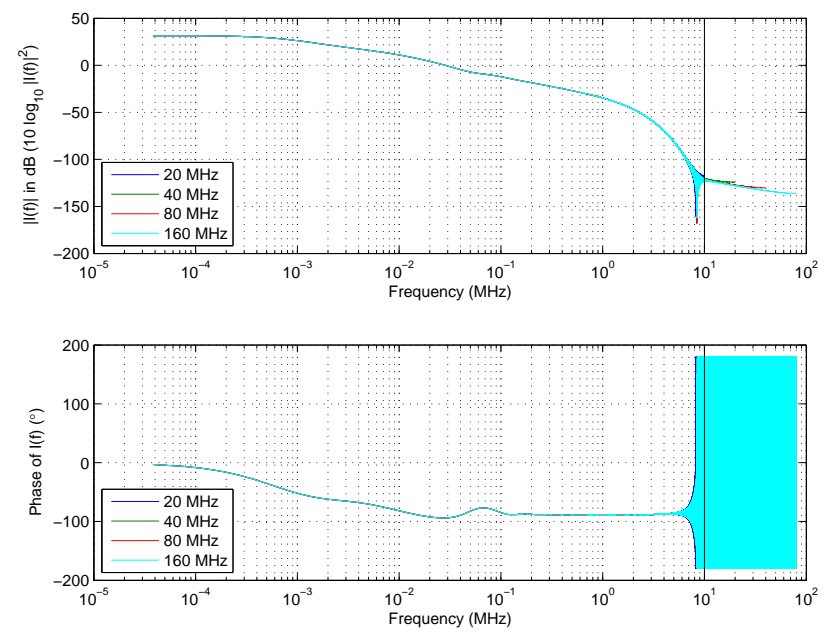

Figure 15: Convergence of the magnitude and phase of the approximate transform of the $1 \%$ Heidler first return stroke current for $T=2 \mathrm{~ms}$. The wrapped phase is taken after multiplying by $\exp (j 2 \pi f \cdot 10 \mu s)$, which corresponds to a time advance of $10 \mu \mathrm{s}$. The vertical solid black line marks $10 \mathrm{MHz}$, which is the highest frequency of interest. 

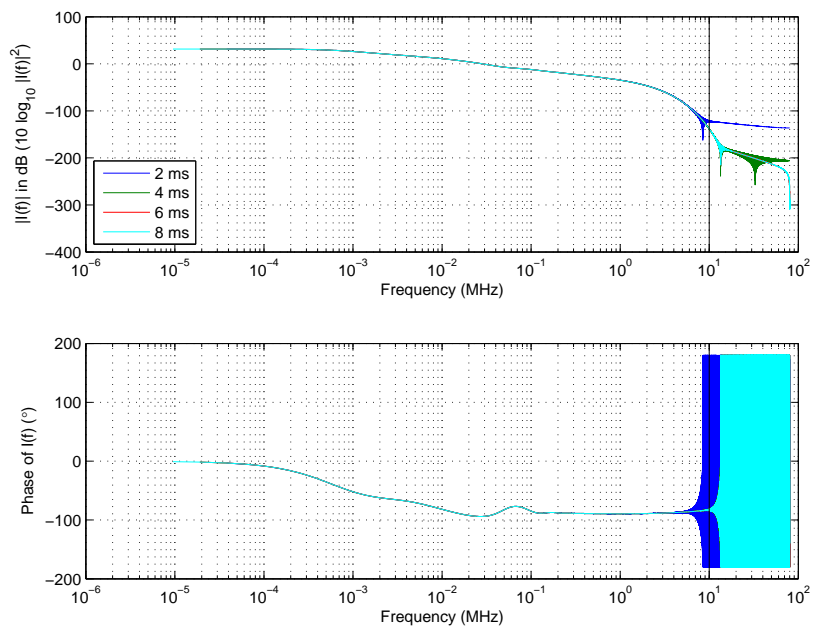

Figure 16: Convergence of the magnitude and phase of the approximate transform of the $1 \%$ Heidler first return stroke current for $f_{s}=160 \mathrm{MHz}$. The wrapped phase is taken after multiplying by $\exp (j 2 \pi f \cdot 10 \mu s)$, which corresponds to a time advance of $10 \mu \mathrm{s}$. The vertical solid black line marks $10 \mathrm{MHz}$, which is the highest frequency of interest.
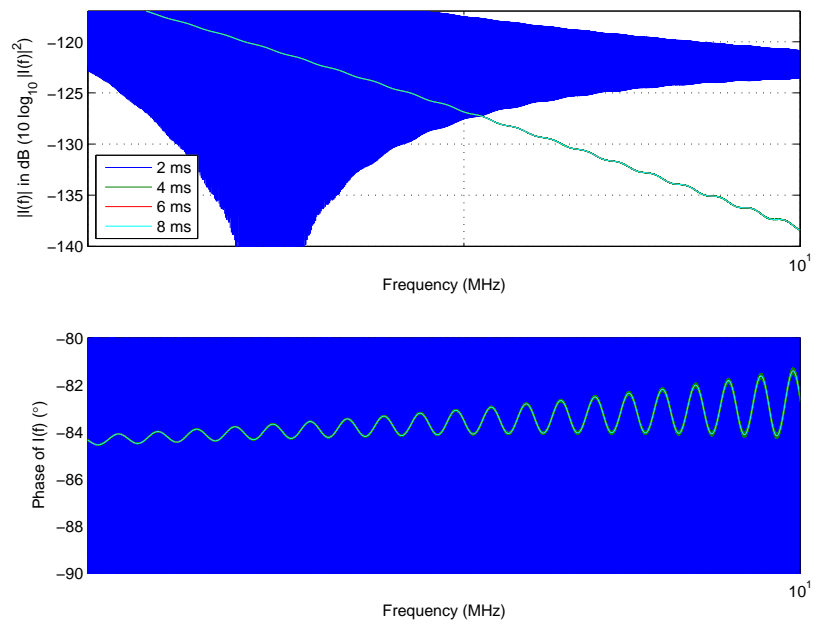

Figure 17: Expanded view of Fig. 16 around $10 \mathrm{MHz}$. 

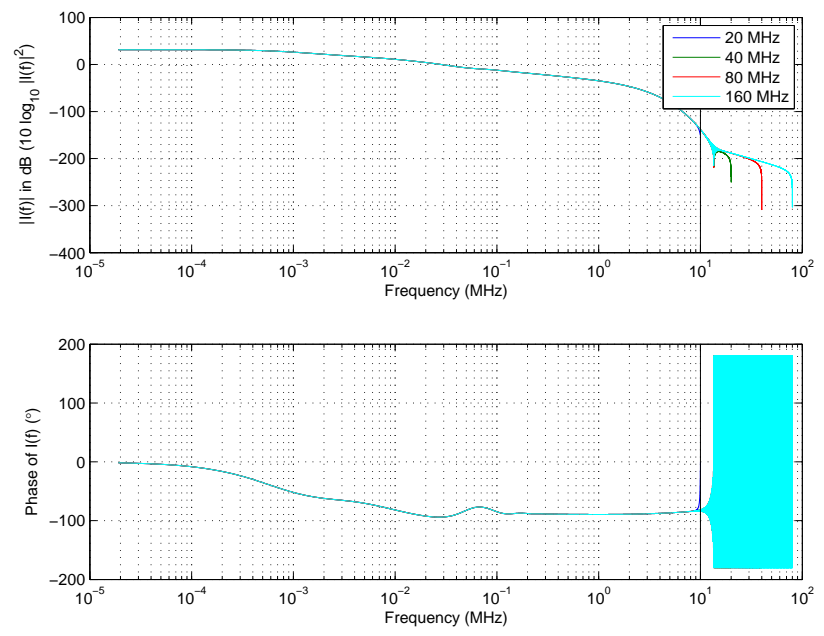

Figure 18: Convergence of the magnitude and phase of the approximate transform of the $1 \%$ Heidler first return stroke current for $T=6 \mathrm{~ms}$. The wrapped phase is taken after multiplying by $\exp (j 2 \pi f \cdot 10 \mu s)$, which corresponds to a time advance of $10 \mu \mathrm{s}$. The vertical solid black line marks $10 \mathrm{MHz}$, which is the highest frequency of interest.
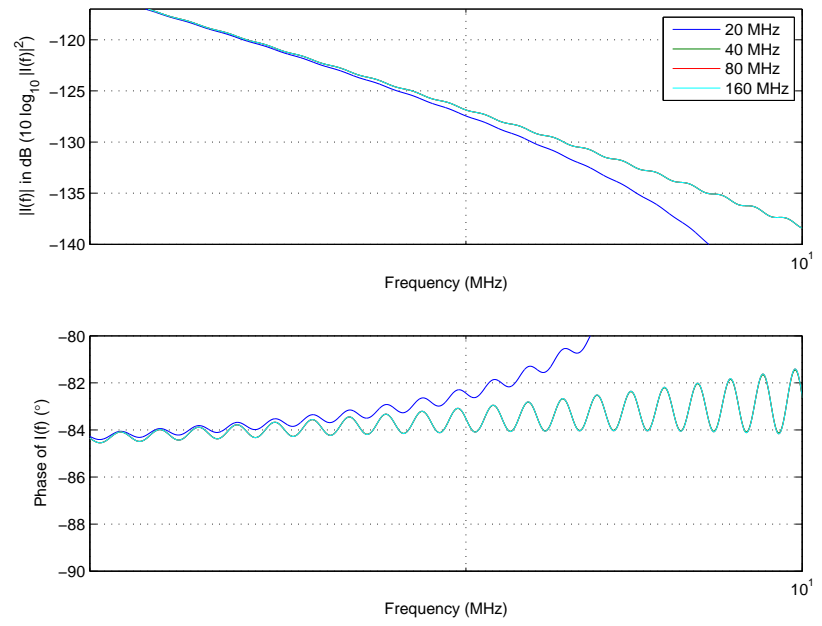

Figure 19: Expanded view of Fig. 18 around $10 \mathrm{MHz}$. 

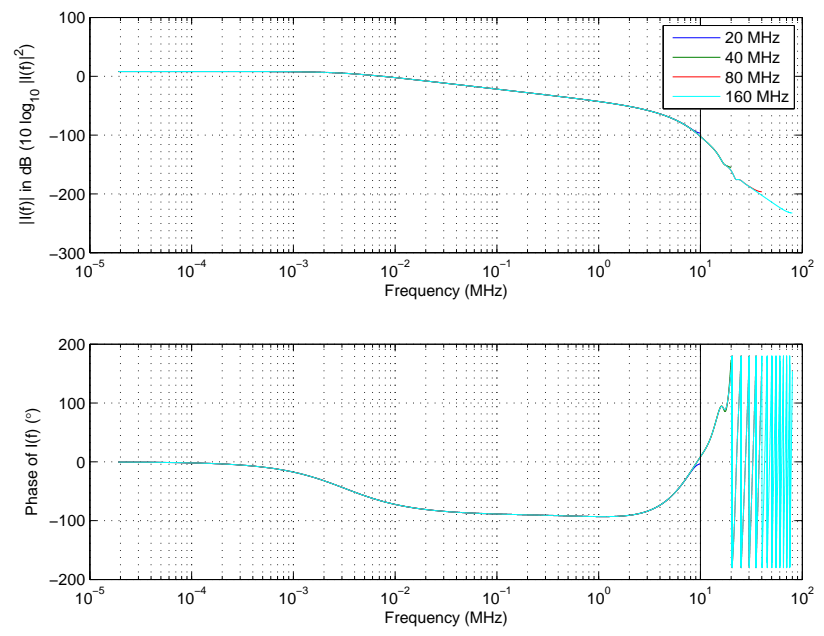

Figure 20: Convergence of the magnitude and phase of the approximate transform of the $1 \%$ Heidler subsequent return stroke current for $T=6 \mathrm{~ms}$. The wrapped phase is taken after multiplying by $\exp (j 2 \pi f \cdot 0.1991 \mu s)($ a $0.1991 \mu \mathrm{s}$ time advance) is plotted. The vertical solid black line marks $10 \mathrm{MHz}$, which is the highest frequency of interest.
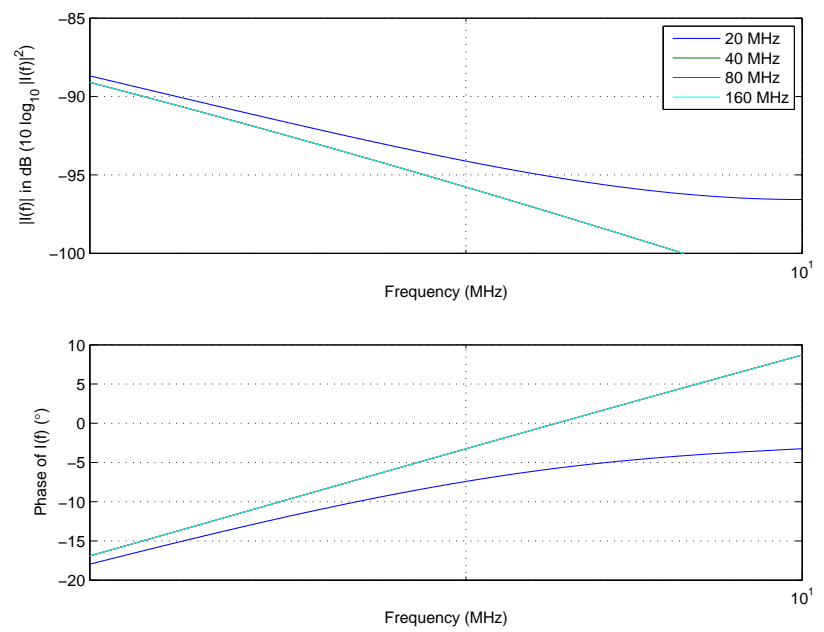

Figure 21: Expanded view of Fig. 20 around $10 \mathrm{MHz}$. 

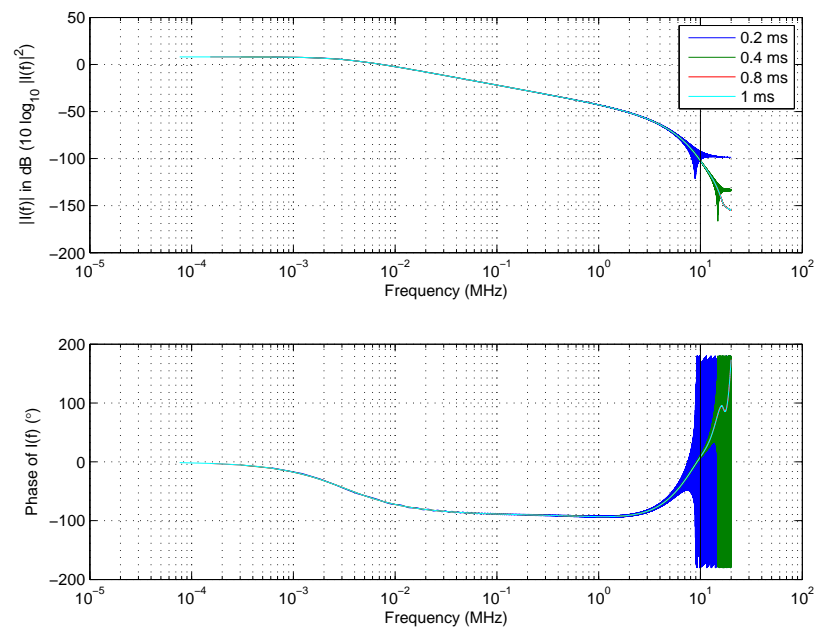

Figure 22: Convergence of the magnitude and phase of the approximate transform of the $1 \%$ Heidler subsequent return stroke current for $f_{s}=40 \mathrm{MHz}$. The wrapped phase is taken after multiplying by $\exp (j 2 \pi f \cdot 0.1991 \mu s)($ a $0.1991 \mu \mathrm{s}$ time advance) is plotted. The vertical solid black line marks $10 \mathrm{MHz}$, which is the highest frequency of interest.
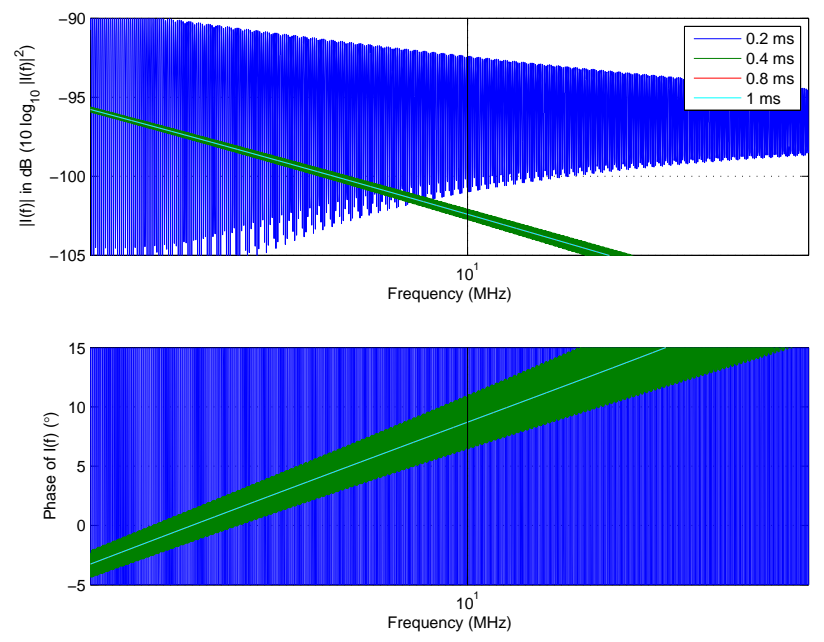

Figure 23: Expanded view of Fig. 22 around $10 \mathrm{MHz}$. 

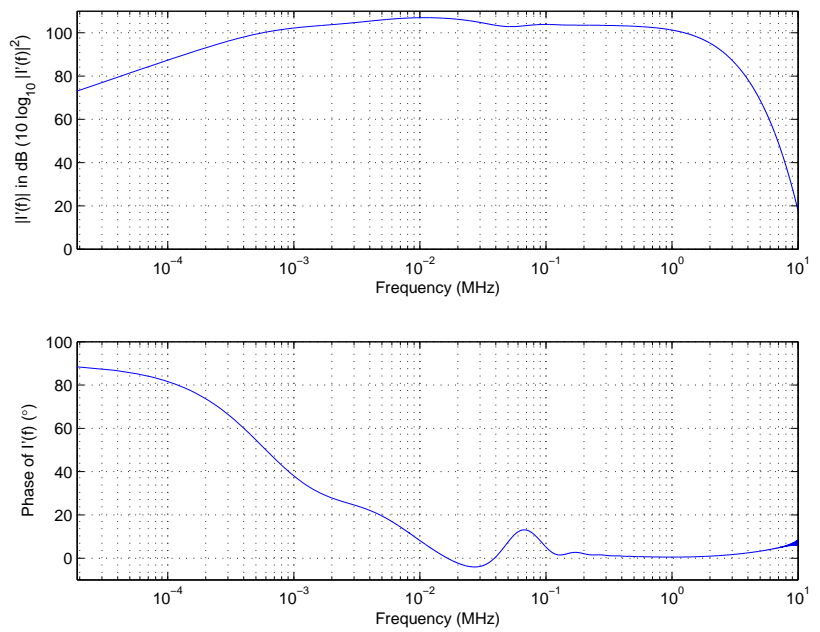

Figure 24: Magnitude and phase of the approximate transform of the first derivative of the $1 \%$ Heidler first return stroke current for $f_{s}=40 \mathrm{MHz}$ and $T=6 \mathrm{~ms}$. The wrapped phase is taken after multiplying by $\exp (j 2 \pi f \cdot 10 \mu s)$, which corresponds to a time advance of $10 \mu \mathrm{s}$.

Finally, we plot the spectra of the $1 \%$ Heidler return stroke currents and first derivatives of the currents with the analytical spectra of the $1 \%$ doubleexponential current and first derivative. Figures 26 and 27 display the magnitude and phase of the $1 \%$ Heidler return stroke currents and the $1 \%$ doubleexponential current. The unwrapped phase of the $1 \%$ Heidler first and subsequent return stroke currents is taken after multiplying by $\exp (j 2 \pi f \cdot 10 \mu s)$ or $\exp (j 2 \pi f \cdot 0.1991 \mu s)$, respectively. Figures 28 and 29 display the magnitude and phase of the first derivative of the $1 \%$ Heidler return stroke currents and of the $1 \%$ double-exponential current. Again, the unwrapped phase of the derivative of the $1 \%$ Heidler first and subsequent return stroke currents is taken after multiplying by $\exp (j 2 \pi f \cdot 10 \mu s)$ or $\exp (j 2 \pi f \cdot 0.1991 \mu s)$, respectively. Table 4 lists the parameters values for $f_{s}$ and $T$ used in Figs. 26, 27, 28, 29, and 30 for the $1 \%$ Heidler cases. The analytical transforms, Eqns. 15 and 16, are used for the $1 \%$ double-exponential current and derivative. A further comparison of the 1\% double-exponential and Heidler cases is in Fig. 30. Figure 30 displays the ratio of the magnitudes of the spectra of the Heidler and double-exponential currents. The ratio of the magnitudes of the spectra of the corresponding first derivatives is identical to Fig. 30, since the $2 \pi f$ terms in the numerator and the denominator cancel. 

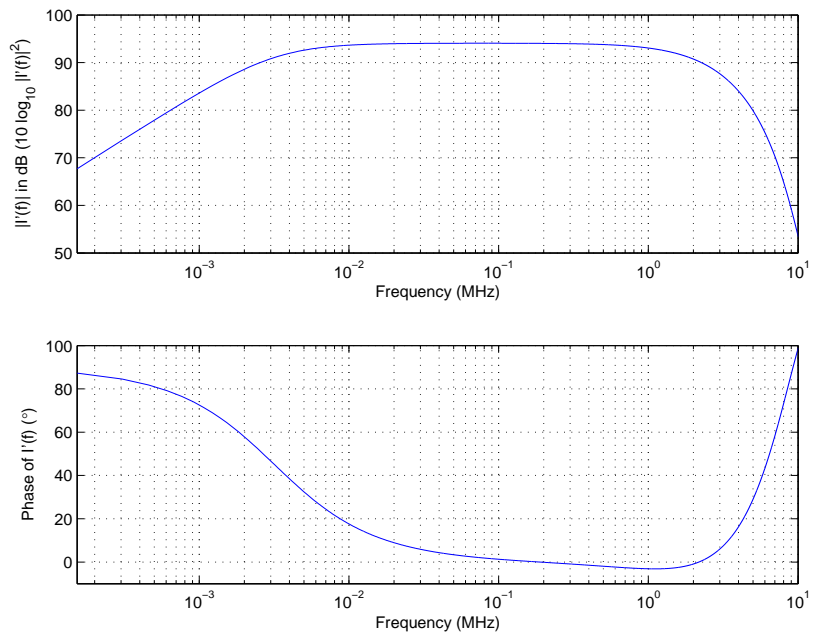

Figure 25: Magnitude and phase of the approximate transform of the first derivative of the $1 \%$ Heidler subsequent return stroke current for $f_{s}=40 \mathrm{MHz}$ and $T=$ $0.8 \mathrm{~ms}$. The wrapped phase is taken after multiplying by $\exp (j 2 \pi f \cdot 0.1991 \mu s)$ (a $0.1991 \mu$ s time advance) is plotted.

Table 4: Parameter values for approximate transforms in Figs. 26, 27, 28, 29, and 30 .

\begin{tabular}{lll}
\hline & $f_{s}(\mathrm{MHz})$ & $T(\mathrm{~ms})$ \\
\hline $\begin{array}{l}1 \% \text { Heidler first return } \\
\text { stroke current and derivative }\end{array}$ & 40 & 6 \\
\hline $\begin{array}{l}1 \% \text { Heidler subsequent return } \\
\text { stroke current and derivative }\end{array}$ & 40 & 0.8 \\
\hline
\end{tabular}




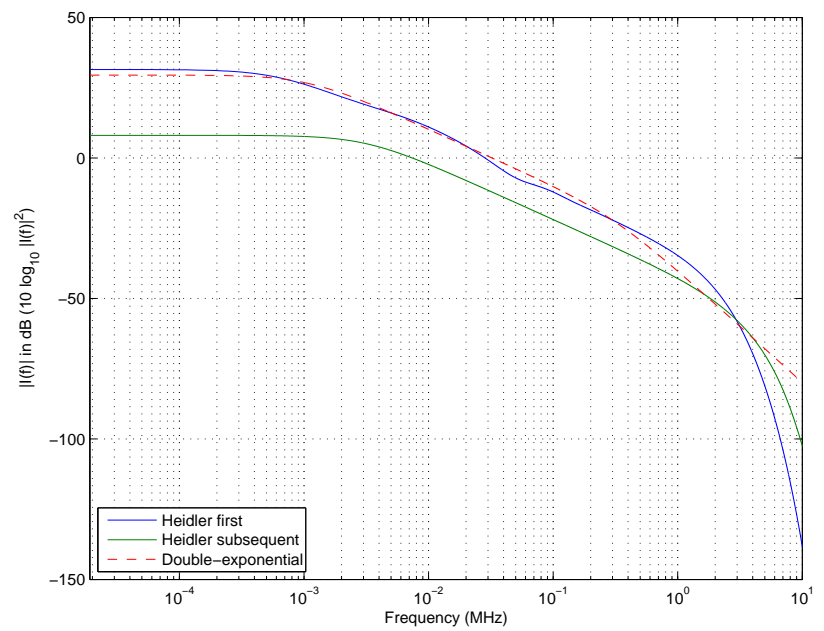

Figure 26: Magnitude of the spectra of the $1 \%$ Heidler return stroke currents, with $f_{s}$ and $T$ from Table 4 , and the analytical spectrum of the $1 \%$ doubleexponential current.

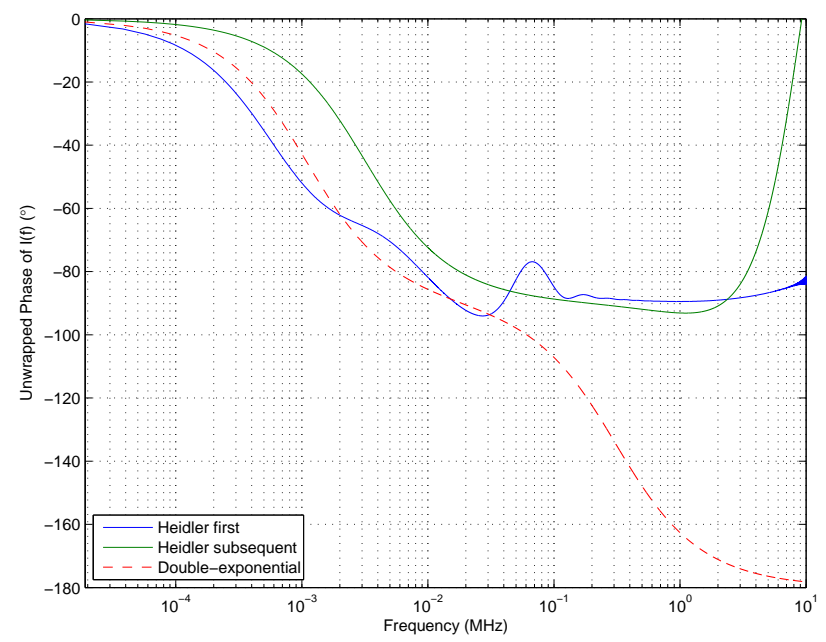

Figure 27: Unwrapped phase of the spectra of the 1\% Heidler return stroke currents, with $f_{s}$ and $T$ from Table 4 , and the analytical spectrum of the $1 \%$ double-exponential current. The unwrapped phase of the spectra of the $1 \%$ Heidler first and subsequent return stroke currents is taken after multiplying by $\exp (j 2 \pi f \cdot 10 \mu s)$ or $\exp (j 2 \pi f \cdot 0.1991 \mu s)$, respectively. 


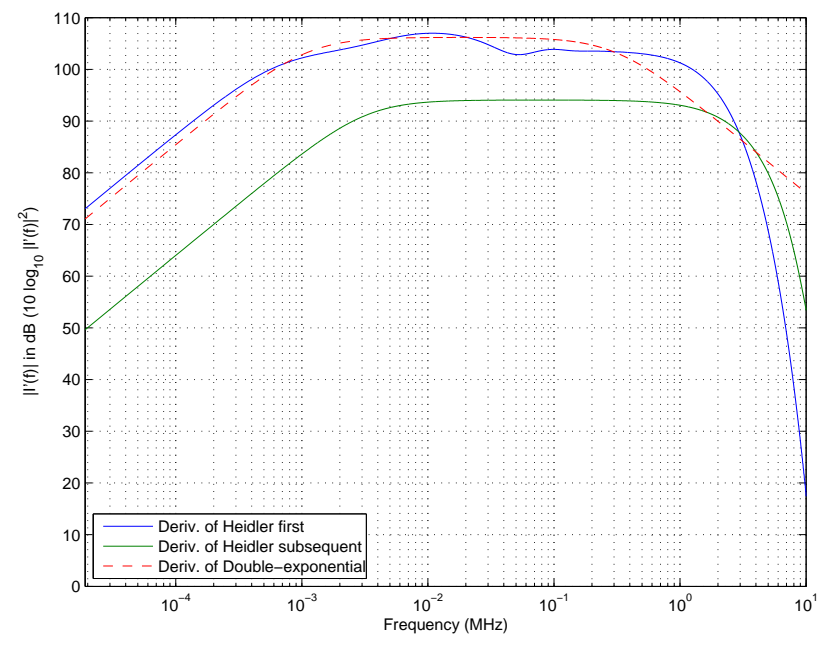

Figure 28: Magnitude of the spectra of the derivative of the $1 \%$ Heidler return stroke currents, with $f_{s}$ and $T$ from Table 4 , and the analytical spectrum of the $1 \%$ double-exponential current.

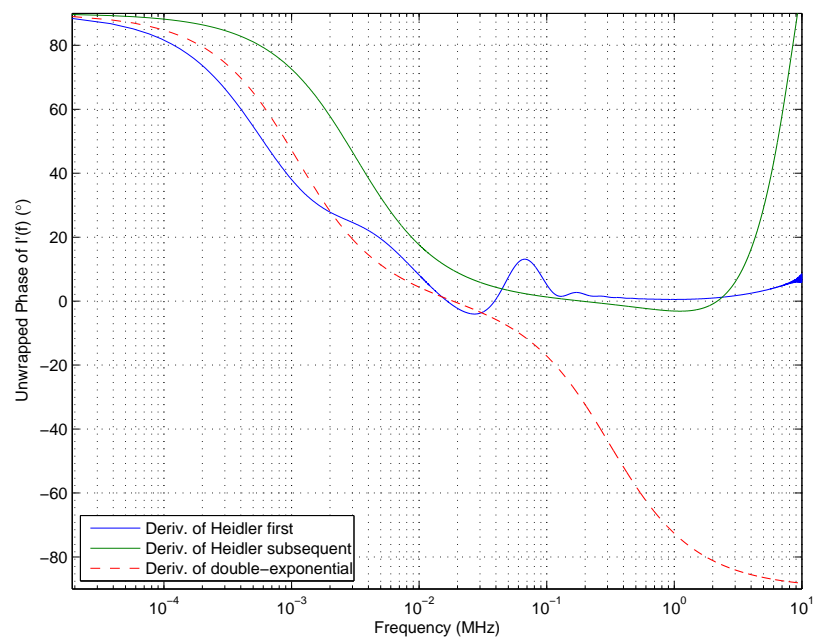

Figure 29: Unwrapped phase of the spectra of the derivative of the $1 \%$ Heidler return stroke currents, with $f_{s}$ and $T$ from Table 4 , and the analytical spectrum of the $1 \%$ double-exponential current. The unwrapped phase of the spectra of the $1 \%$ Heidler first and subsequent return stroke currents is taken after multiplying by $\exp (j 2 \pi f \cdot 10 \mu s)$ or $\exp (j 2 \pi f \cdot 0.1991 \mu s)$, respectively. 


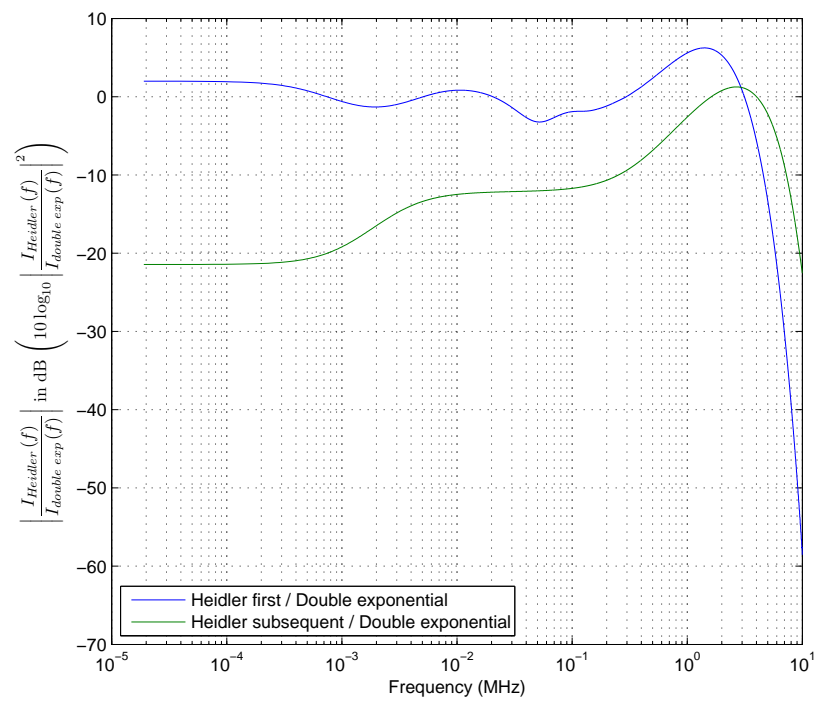

Figure 30: Ratio of the magnitudes of the spectra of the Heidler currents, with $f_{s}$ and $T$ from Table 4, and the analytical spectrum of the double-exponential current. The ratio of the magnitudes of the spectra of the corresponding first derivatives is identical to Fig. 30, since the $2 \pi f$ terms in the numerator and the denominator cancel.

\section{References}

[1] M. A. Uman, V. A. Rakov, J. O. Elisme, D. M. Jordan, C. Biagi, and J. D. Hill, "Update direct-strike lightning environment for stockpile-to-target sequence," Tech. Rep. LLNL-SR-407603, Lawrence Livermore National Laboratory, September 2008.

[2] R. J. Fisher and M. A. Uman, "Recommended baseline direct-strike lightning environment for stockpile-to-target sequence," Tech. Rep. SAND890192 UC-13, Sandia National Laboratory, May 1989.

[3] S. W. Smith, Digital Signal Processing: A Practical Guide for Engineers and Scientists. Newnes, 2003.

[4] L. W. Couch II, Digital and Analog Communication Systems. Prentice Hall, 1997.

[5] J. V. Candy, Signal Processing: The Modern Approach. McGraw-Hill, 1988.

[6] E. Brigham, Fast Fourier transform and its applications. 1988. 
[7] E. A. Morelli, "High accuracy evaluation of the finite fourier transform using sampled data," Tech. Rep. 110340, NASA Langley Research Center, June 1997.

[8] A. V. Oppenheim and R. W. Schafer, Discrete-Time Signal Processing. Prentice Hall, 1989.

[9] Discrete Fourier Transform: the real-input DFT. http://en.wikipedia.org/wiki/Discrete_Fourier_transform\#The_realinput_DFT (accessed October 2009).

[10] C. Buccella, S. Cristina, and A. Orlandi, "Frequency analysis of the induced effects due to the lightning stroke radiated electromagnetic field," IEEE Transactions on Electromagnetic Compatibility, vol. 34, pp. 338-44, August 1992. 